\title{
Anspruch auf Änderung des Arbeitspensums
}

\section{Anregungen zur Stärkung der Zeitsouveränität von Arbeitnehmenden}

\section{Sabine Steiger-Sackmann *}

Arbeitnehmende in privatrechtlichen Arbeitsverhältnissen riskieren in der Schweiz den Verlust ihrer Stelle, wenn sie ihr Arbeitspensum reduzieren wollen. Für Arbeitskräfte in Deutschland und den Niederlanden besteht hingegen ein gesetzlicher Anspruch auf Verringerung der Arbeitszeit, und in einigen Ländern müssen Mitarbeitende bei Stellenbesetzungen bevorzugt werden, wenn sie von einem Teilzeit- in ein Vollzeitpensum wechseln wollen. Einige Ansätze für eine stärkere Berücksichtigung der Arbeitszeitwünsche von Arbeitnehmenden sind auch in der Schweiz zu verzeichnen; es gibt neben sozialpolitischen auch rechtliche Gründe, diese weiter zu verfolgen.

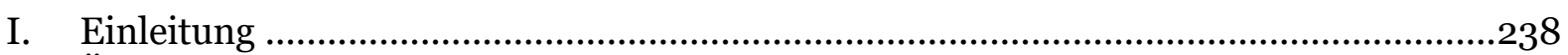

II. Änderung des Arbeitspensums nach geltendem Recht .................................................239

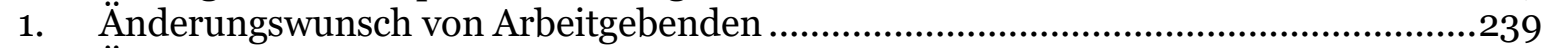

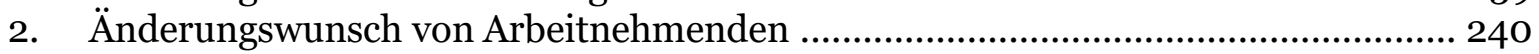

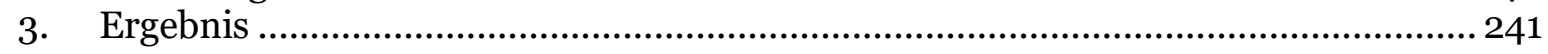

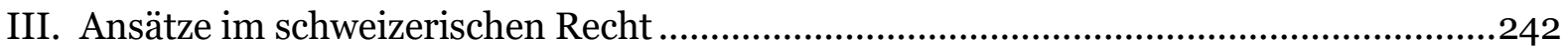

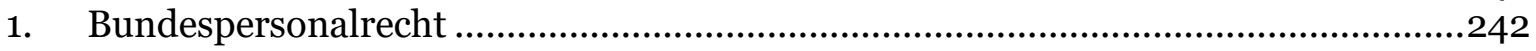

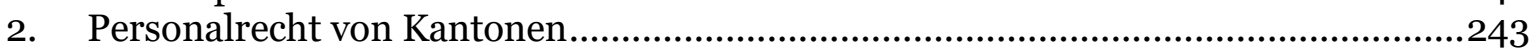

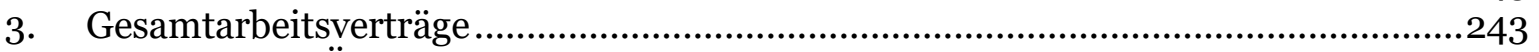

4. Vorstösse zur Änderung des Obligationenrechts......................................................244

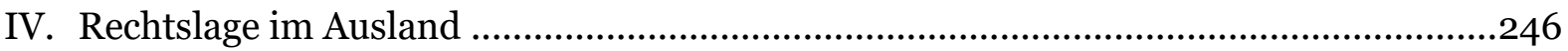

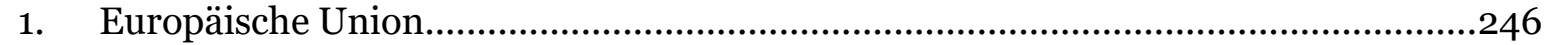

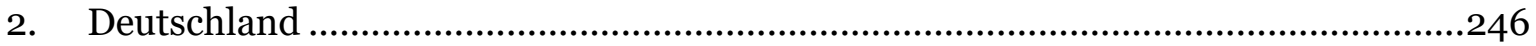

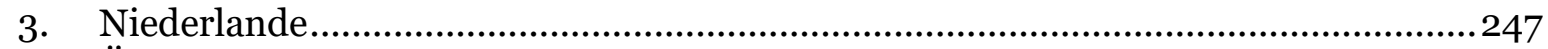

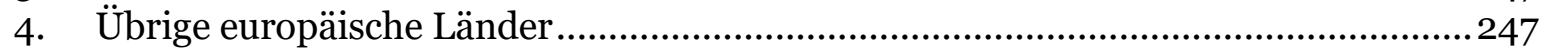

5. Erfahrungen mit Anpassungsansprüchen ...............................................................249

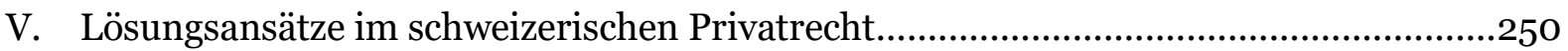

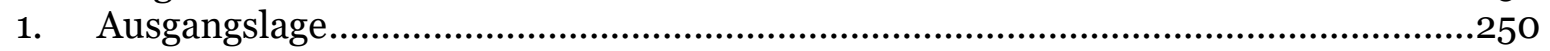

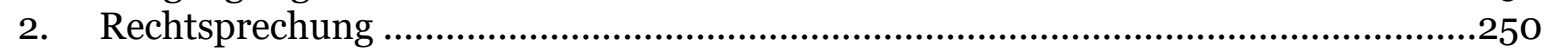

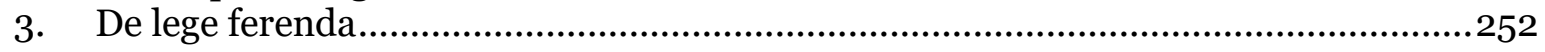

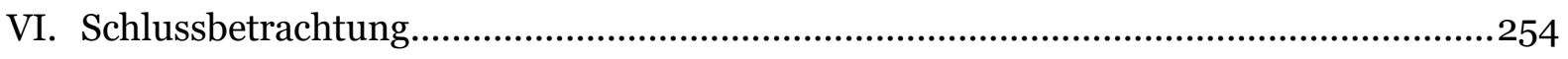

Zitiervorschlag: Sabine Steiger-Sackmann, Anspruch auf Änderung des Arbeitspensums: Anregungen zur Stärkung der Zeitsouveränität von Arbeitnehmenden, in: sui-generis 2018, S. 237

URL: $\quad$ sui-generis.ch/71

DOI: $\quad$ https://doi.org/10.21257/sg.71

* Sabine Steiger-Sackmann (stsa[at]zhaw.ch), Dr. iur., Dozentin für Arbeits- und Sozialversicherungsrecht an der Zürcher Hochschule für Angewandte Wissenschaften. 


\section{Einleitung}

1 Beim Abschluss eines Arbeitsvertrags müssen sich die Parteien darüber einigen, ob die Arbeitsleistung im betriebsüblichen Umfang als Vollzeitpensum oder als Teilzeitpensum in einem bestimmten Umfang zu erbringen ist. Dementsprechend werden sie auch den Lohn festsetzen. Als Dauerschuldverhältnis muss das Arbeitsverhältnis aber für die Zukunft offen sein. Daher anerkennt das Recht, dass Anpassungen von einmal getroffenen Vereinbarungen möglich sind. Solange sich beide Parteien über eine Änderung des Arbeitspensums und den Zeitpunkt der Umsetzung einig sind, wirft dies rechtlich keine Probleme auf ${ }^{1}$.

2 Der Wunsch nach einer Erhöhung oder Reduktion des Pensums wird von betrieblicher Seite in der Regel wirtschaftlich begründet, weil das Arbeitsvolumen nicht mehr mit dem Personalbestand übereinstimmt. Arbeitnehmende streben eine Erhöhung des Arbeitspensums ebenfalls meist aus wirtschaftlichen Gründen (Erhöhung des Lohnes) an. Eine Reduktion des Pensums wird jedoch nicht in erster Linie wegen geringerem Einkommensbedarf gewünscht; vielmehr hängt der Wunsch nach Reduktion der Erwerbsarbeit meistens mit familiärer Betreuungsarbeit oder persönlichen Bedürfnissen (wie Bildung oder Gesundheit) zusammen². Da die durchschnittli-

\footnotetext{
Aurélien Witzig, La modification du rapport du travail, Genf 2014, S. $112 \mathrm{ff}$.

2 Als Gründe für eine Teilzeitbeschäftigung werden hauptsächlich familiäre Gründe genannt (42,3\%; $15,9 \%$ bei Männern und 49,5 \% bei Frauen); weitere Gründe sind Absolvieren einer Ausbildung $(14,2 \%)$, erfolglose Suche nach einer Vollzeitarbeit (10,8\%), Ausüben einer Nebentätigkeit $(6,9 \%)$ und gesundheitliche Gründe (3,8\%). Ausserdem gaben $10,8 \%$ an, kein Interesse an einer Vollzeiterwerbstätigkeit $\mathrm{zu}$ haben, und
}

che Arbeitszeit in der Schweiz mit 41,1 Wochenstunden 3 im Vergleich zu andern europäischen Staaten hoch ist 4 , besteht hierzulande eher ein Bedürfnis nach einem Teilzeitpensum.

3 Es wird zu Recht darauf hingewiesen, dass die soziale Absicherung bei Teilzeiterwerbstätigkeit im Vergleich mit einer vollen Erwerbstätigkeit schlechter ist 5 und freiwillige Pensumsreduktionen zum Verlust von Ansprüchen führen können6. Den Arbeitnehmenden, die dennoch eine Reduktion der Arbeitszeit wünschen, sollten aber aus vertragsrechtlicher Sicht keine unnötigen Steine in den Weg gelegt werden.

4 Enthält ein synallagmatischer Vertrag keine Regelung für Anpassungen und können sich die Parteien nicht darüber einigen, kommen gesetzliche oder richterrechtliche Grundlagen zur Anwendung. Diese zielen darauf ab, die rechtsgeschäftlich begründete Werterelation wiederherzustellen bzw. das Änderungs-

13,2\% führten andere Gründe an; Bundesamt für Statistik, SAKE in Kürze 2016, Neuenburg 2017, S. 16.

3 Bundesamt für Statistik, Tatsächliche Jahresarbeitszeit und tatsächliche wöchentliche Arbeitszeit der Vollzeiterwerbstätigen 2017, 24. Mai 2018.

4 Sechste Europäische Erhebung über die Arbeitsbedingungen.

5 Viele Leistungen der Sozialversicherungen knüpfen an den früheren Lohn an, und die Versicherung in der beruflichen Vorsorge ist erst ab einer lohnmässigen Schwelle obligatorisch. Ausführlich dazu Gilles Brugger, Die Arbeitsverhinderung bei Teilzeiterwerbstätigen und Mehrfachbeschäftigten, Diss. St. Gallen, Zürich 2017.

6 Berufsvorsorgerechtlich kann bei der Frage nach zeitlicher Konnexität von Bedeutung sein, ob eine Pensumsreduktion krankheitsbedingt oder freiwillig erfolgt ist (Urteil des Bundesgerichts 9C_653/2016 vom 2. März 2017, E. 4.3). 
risiko adäquat zu verteilen7. Der allgemeine Teil des schweizerischen Obligationenrechts enthält keine gesetzliche Regelung über Anpassungsrechte und Anpassungsfolgen ${ }^{8}$. Mit der Rechtsfigur der clausula rebus sic stantibus besteht eine schwache richterrechtliche Rechtsgrundlage 9 . Sie wurde nur in Einzelfällen ins Gesetz übernommen (z.B. beim Werkvertrag Art. 373 Abs. 2 OR ${ }^{10}$ ).

5 Auch die Bestimmungen des Obligationenrechts über den privatrechtlichen Arbeitsvertrag enthalten keine generelle Bestimmung über die Anpassung des Vertrags; aus dem Grundsatz der Vertragsfreiheit wird jedoch eine allgemeine Änderungs- und Beendigungsfreiheit abgeleitet ${ }^{11}$. Bei den Regelungen zur Kündigung des Arbeitsvertrags wurde vom Gesetzgeber Wert auf eine paritätische Ausgestaltung gelegt ${ }^{12}$ (selbst beim Schutz vor missbräuchlicher Kündigung, der nur zum Schutz der Arbeitnehmenden erfor-

7 Hubert Stöckli, Das Synallagma im Vertragsrecht, Begründung - Abwicklung - Störung, Diss. Freiburg, Zürich 2008, Rz. 72.

8 Dies im Unterschied zu Deutschland. § 313 Abs. 1 BGB regelt: «Haben sich Umstände, die zur Grundlage des Vertrags geworden sind, nach Vertragsschluss schwerwiegend verändert ..., so kann Anpassung des Vertrags verlangt werden, soweit einem Teil unter Berücksichtigung aller Umstände des Einzelfalls, insbesondere der vertraglichen oder gesetzlichen Risikoverteilung, das Festhalten am unveränderten Vertrag nicht zugemutet werden kann.»

9 Witzig (Fn. 1), S. 167.

10 Bundesgesetz betreffend die Ergänzung des Schweizerischen Zivilgesetzbuches vom 30. März 1911 (Fünfter Teil: Obligationenrecht, SR 220).

11 Bernhard Stehle, Art. 19 OR, in: Präjudizienbuch OR, Die Rechtsprechung des Bundesgerichts (1875-2015), 9. Aufl., Zürich 2016.

12 Botschaft vom 9. Mai 1984 zur Volksinitiative «betreffend Kündigungsschutz im Arbeitsvertragsrecht» und zur Revision der Bestimmungen über die Auflösung des Arbeitsverhältnisses im Obligationenrecht (BBl 1984 II 551), S. 557. derlich ist ${ }^{13}$ ). Äquivalenz und (wo angezeigt) Parität müssten demzufolge auch in Bezug auf die Durchsetzung von Änderungswünschen der Parteien im Arbeitsvertrag gelten. Dem ist nach aktueller Rechtslage aber nicht so, wie im folgenden Kapitel gezeigt wird.

\section{II. Änderung des Arbeitspensums nach geltendem Recht}

\section{1. Änderungswunsch von Arbeitgebenden}

6 Steigt das Arbeitsvolumen, sind Unternehmen berechtigt Überstunden und u.U. Überzeit anzuordnen. Die Arbeitnehmenden auf der anderen Seite sind verpflichtet, Überstunden zu leisten, soweit ihnen dies zumutbar ist (Art. 321c OR) und die vom Arbeitsgesetz gesetzten Grenzen eingehalten werden (Art. $9 \mathrm{ff}$. ArG'14).

7 Bei voraussichtlich vorübergehender Verschlechterung der Auftragslage können Betriebe Kurzarbeit beantragen, damit sie Arbeitsverträge nicht kündigen oder Arbeitspensen von einzelnen Mitarbeitenden nicht mittels Änderungskündigung15 kürzen müssen (Art. $31 \mathrm{ff}$. AVIG $\left.{ }^{16}\right)$. Bei längerfristig reduziertem Personalbedarf können Unternehmen entscheiden, ob sie die verbleibende Ar-

13 Alexandre Berenstein, La «protection paritaire contre le congé» dans le contrat de travail, in: SJZ 80 (1984), S. 112.

14 Bundesgesetz über die Arbeit in Industrie, Gewerbe und Handel vom 13. März 1964 (Arbeitsgesetz, SR 822.11).

15 Ullin Streiff/Adrian von Kaenel/Roger Rudolph, Arbeitsvertrag, Praxiskommentar zu Art. 319-362 OR, 7. Aufl., Zürich 2012, Art. 335 OR N 3.

16 Bundesgesetz über die obligatorische Arbeitslosenversicherung und die Insolvenzentschädigung vom 25. Juni 1982 (Arbeitslosenversicherungsgesetz, SR 837.0). 
beit nur mit Vollzeit- oder auch mit Teilzeitkräften ausführen wollen. Werden die Bestimmungen über Massenentlassungen eingehalten (Art. 335d ff. OR) und erweisen sich solche Kündigungen nicht aus einem anderen Grund als missbräuchlich (Art. 336 OR), können sich die Arbeitnehmenden nicht dagegen wehren. Wollen sie das Angebot eines reduzierten Pensums nicht hinnehmen ${ }^{17}$, weil sie auf das volle Einkommen angewiesen sind, aber noch keine Ersatzstelle gefunden haben, kann durch die Arbeitslosenversicherung die Anspruchsberechtigung für Taggelder wegen selbstverschuldeter Arbeitslosigkeit für gewisse Zeit eingestellt werden (Art. 30 Abs. 1 lit. a AVIG).

\section{2. Änderungswunsch von Arbeitnehmenden}

8 Äussern Arbeitnehmende den Wunsch, ihr Arbeitspensum zu erhöhen, haben sie kein Recht, bei einem Ausbau des Personalbestands berücksichtigt zu werden.

9 Arbeitgebende haben nach heutiger Rechtslage in privatrechtlichen Verhältnissen auch keine rechtliche Verpflichtung, Wünschen ihrer Mitarbeitenden nach einer Pensumsreduktion nachzukommen oder die Ablehnung solcher Wünsche zu begründen. Sind Arbeitnehmende kurzfristig an der Arbeitsleistung verhindert, sind sie je nach Grund für die Verhinderung vorübergehend von der Arbeitspflicht entbunden (Art. 329 OR; Art. 35a und 36 ArG), und haben vielleicht noch einen Lohnfortzahlungs-

$17 \overline{\text { Auch bei zu langen Abklärungen droht Arbeit- }}$ nehmenden bei einer Änderungskündigung, dass die Kündigung infolge Ablauf der Bedenkfrist wirksam wird und dies u.U. zu Einstelltagen führen kann (Urteil des Bundesgerichts 8C_872/ 2011 vom 6. Juni 2012, ARV 2012, S. 294). anspruch (Art. 324a OR) ${ }^{18}$. Können sie das bisherige Arbeitspensum aber auf längere Sicht nicht mehr erbringen und war keine einvernehmliche Reduktion des Arbeitspensums zu erreichen, bleibt den Betroffenen nichts anderes übrig, als die bisherige Stelle selber zu kündigen und sich auf die Suche nach einer passenden Teilzeitstelle zu begeben. Wenn sie eine verhältnismässig gleich gut bezahlte Teilzeitstelle gefunden haben, sind sie während der Probezeit und der Karenzzeit für die Lohnfortzahlung (Art. 324a Abs. 1 OR) sowie bezüglich Dienstjahren in einer rechtlich bedeutend schlechteren Position als im alten Arbeitsverhältnis.

10 Kündigt die Arbeitgeberin nach der Ablehnung eines Änderungswunsches aus familiären Gründen das Arbeitsverhältnis, muss sie allenfalls damit rechnen, dass Betroffene die Kündigung als diskriminierend gem. Art. 9 GlG 19 anfechten. Arbeitnehmende, die aus einem anderen Grund das Pensum ändern wollten, können sich gegen eine Kündigung aber nicht wehren mit der Begründung, sie hätten nach Treu und Glauben ein Recht geltend gemacht (Rachekündigung gem. Art. 336 Abs. 1 lit. d OR), denn in der Schweiz besteht kein Recht auf Anpassung des Arbeitspensums. Selbst in diesen Fällen ist der Rechtsnachteil für Unternehmen limitiert auf eine Entschädigung im Umfang von i.d.R. zwei bis drei Monatslöhnen. Die Gekündigten haben in keinem Fall einen Anspruch auf Weiterbeschäftigung. Erträgt aber z.B.

18 Ausführlich dazu: Angela Hensch, Arbeitnehmer mit Familienpflichten, in: AJP 2016, S. 1631$1644,1634 \mathrm{ff}$.

19 Bundesgesetz über die Gleichstellung von Frau und Mann vom 24. März 1995 (Gleichstellungsgesetz, SR 151.1). 
die Betreuungsaufgabe, wegen welcher Beschäftigte das Pensum reduzieren wollen, keinen Aufschub und können sie mit der Kündigung nicht zuwarten, bis sie eine geeignete Stelle gefunden haben, drohen ihnen wiederum Einstelltage bei der Arbeitslosenkasse ${ }^{20}$.

11 Einer anderen rechtlichen Beurteilung bedarf aber der folgende Fall: Eine alleinstehende Sekretärin mit dauerhaft zu hoher Arbeitslast möchte ihr Pensum reduzieren, um gesund zu bleiben. Eine solche Meldung muss das Unternehmen dazu veranlassen, die Arbeitslast auf ein gesundheitsverträgliches Niveau anzupassen, sofern es sich nicht um tätigkeitsimmanente, unvermeidbare Belastungen handelt (Art. 328 OR bzw. Art. 6 ArG), denn Unternehmen können nicht die Präventionspflichten vernachlässigen in der Hoffnung, dass ihnen nur noch die robustesten Arbeitnehmenden bleiben. Spätestens wenn bekannt ist ${ }^{21}$, dass die Arbeitslast die Gesundheit einer Mitarbeiterin beeinträchtigt, müssen die geeigneten, erforderlichen und zumutbaren Massnahmen zum Schutz der Gesundheit getroffen werden. Wenn dennoch aus Sicht der Betroffenen die Reduktion des Pensums angezeigt ist (und sie erst noch die daraus resultierende Lohneinbusse in Kauf nimmt), haftet ein Unternehmen, welches diesen Wunsch abschlägt, für allfällige Folgen ${ }^{22}$.

$2 0 \longdiv { \text { Hier müsste dann allerdings geprüft werden, ob } }$ der Verbleib in der früheren Stelle zumutbar gewesen wäre (Art. 44 Abs. 1 lit. b Arbeitslosenversicherungsverordnung vom 31. August 1983, AVIV; SR 837.02).

21 Urteil des Bundesverwaltungsgerichts A-4147/ 2016 vom 4. August 2017, E. 7.7.

22 Ausführlich dazu: Sabine Steiger-Sackmann, Grundlagen und Tücken von Stresshaftungsklagen, in: AJP 6/2017, S. 729-738.

\section{Ergebnis}

12 Arbeitnehmende haben ein hohes Risiko zu tragen, wenn sie ihre Änderungswünsche bezüglich des Arbeitspensums umsetzen wollen. Sie riskieren, einen Teil ihrer Einkommensbasis zu verlieren, und sind u.U. existenziell betroffen. Häufig beruht ihr Wunsch nach Teilzeitarbeit nicht auf einer völlig freien Entscheidung, sondern auf sozialen Zwangslagen, wie Betreuung von Kindern und kranken Angehörigen. Auch eine Ausbildung oder die Übernahme von Ämtern im Milizsystem sind Gründe, die gesellschaftlich relevant sind, so dass es schwer zu rechtfertigen ist, dass diesen Arbeitnehmenden das Risiko eines Stellenverlustes mit allen damit verbundenen Nachteilen aufgebürdet wird. Lehnen Arbeitgebende einen Reduktionswunsch ab und können Arbeitnehmende deswegen sozial erwünschte Tätigkeiten nicht ausführen, fallen gegebenenfalls Kosten an, an denen sich Arbeitgebende aber nicht zu beteiligen brauchen.

13 Auf der anderen Seite ermöglicht die schweizerische Rechtsordnung den Arbeitgebenden in grossem Umfang, ihre Veränderungsbedürfnisse nach oben und nach unten umzusetzen, auch in Situationen, in denen es noch keineswegs um die Existenz eines Betriebes geht. Die Beschäftigung von Teilzeit-Arbeitnehmenden ist freilich nicht per se nachteilig für die Unternehmen ${ }^{23}$. Trotz etwas höheren Rekrutierungs- und Fixkosten und erhöhtem Organisationsaufwand bringt sie

23 Die Verteilung der Arbeitslast auf mehrere Schultern vermindert das Risiko, dass durch Krankheitsabsenzen der Geschäftsgang infolge Knowhow-Lücken erheblich beeinträchtigt wird (Urteil des Verwaltungsgerichtts ZH VB.2017.00123 vom 3. Oktober 2017, E. 3.4.). Dazu auch Brugger (Fn. 5), Rz. 49 ff. 
mehr Flexibilität bezüglich Arbeitsvolumen und ermöglicht Produktivitätsgewinne durch produktiveres Personal und eine bessere Auslastung der Geräteinfrastruktur ${ }^{24}$.

14 Von tatsächlicher Vertrags(änderungs)freiheit und Kündigungsparität kann nach geltendem Privatrecht nicht die Rede sein; schon gar nicht von einem Recht auf eine selbstbestimmte Erwerbsbiographie $^{25}$ oder von lebensphasenorientierter Zeitsouveränität ${ }^{26}$. Zur Herstellung gleicher Handlungsspielräume bei Veränderungswünschen sind daher rechtliche Ausgleichsmassnahmen geboten. Dazu gibt es sowohl in der Schweiz wie auch im Ausland bereits Ansätze, die es zu untersuchen und zu diskutieren lohnt.

\section{Ansätze im schweizerischen Recht}

\section{Bundespersonalrecht}

15 Gemäss Art. 64 Abs. 4 der Bundespersonalverordnung 27 werden den Angestellten der Bundesverwaltung, soweit es betrieblich möglich ist, flexible Arbeitszeitmodelle sowie die Möglichkeit zur Teilzeitarbeit und zum Jobsharing angeboten.

24 Bundesamt für Statistik, Teilzeitarbeit in der Schweiz, Neuenburg, 2006, S. 22.

25 Dazu ausführlich Eva Kocher/Henning Groskreutz/Ghazaleh Nassibi/Christian Paschke/ Susanne Schulz/Felix Welti/Johanna Wenckebach/Barbara Zimmer, Das Recht auf eine selbstbestimmte Erwerbsbiographie, Arbeits- und sozialrechtliche Regulierungen für Übergänge im Lebenslauf: ein Beitrag zu einem Sozialen Recht der Arbeit, Baden-Baden 2013.

26 Annemarie Aumann/Melanie Hack, Wahlarbeitszeit und Arbeitszeitflexibilisierung; Modelle einer selbstbestimmten Erwerbsbiografie in Deutschland und Norwegen, in Zesar 7/2016, S. 266-276, 276.

27 Bundespersonalverordnung vom 3. Juli 2001 (BPV, SR 172.220.111.3).
16 Konkretisiert wird diese personalpolitische Absichtserklärung mit Art. 60a BPV. Aufgrund dieser Bestimmung haben seit 2013 beide Elternteile nach der Geburt bzw. Adoption eines Kindes einen Anspruch auf eine Reduktion des Beschäftigungsgrades. Die Reduktion muss innerhalb des ersten Jahres nach der Geburt bzw. Adoption beantragt werden. Sie darf maximal 20 Prozent betragen, und der Beschäftigungsgrad darf danach 60 Prozent nicht unterschreiten. Diese Regelung gilt für den Normalfall. Aufgrund der Fürsorgepflicht kann bei einer medizinisch nachgewiesenen Notlage eine weitergehende Reduktion des Pensums angezeigt sein, soweit dem nicht betriebliche Gründe entgegen stehen ${ }^{28}$.

17 Die Erfahrungen in der Bundesverwaltung werden positiv beurteilt. Im Jahr 2014 haben 69 Männer und 70 Frauen vom Rechtsanspruch auf Pensumsreduktion Gebrauch gemacht. In den höheren Lohnklassen waren es gar 41 Männer und 34 Frauen. Der Reduktionsanspruch scheint demnach die bisherige Rollenaufteilung nicht $\mathrm{zu}$ zementieren, sondern hilft vielmehr sie auszugleichen ${ }^{29}$.

18 Mit der Motion «Ein moderner Arbeitgeber; Bundesangestellte sollen ihre Arbeitszeit einfacher reduzieren können» 30 wollte die Grüne Fraktion ferner erreichen, dass die Bundesangestellten ihre Arbeitszeit zumindest einmal in ihrer beruflichen Laufbahn um mindestens 10

28 Urteil des Bundesverwaltungsgerichts A-546/ 2014 vom 16. Juni 2014. Dazu auch Urteilsbesprechung von Philipp Conradin-Triaca in: ZVInfo September 2014, S. 21.

29 Angaben gemäss Votum Nationalrätin Nadine Masshardt am 1. Juni 2017 zum Geschäft Nr. 15.470 (AB 2017 N 881).

30 Gesch.-Nr. 16.3397. 
Prozent reduzieren können, wobei der Beschäftigungsgrad 50 Prozent nicht hätte unterschreiten dürfen. Laut Stellungnahme des Bundesrates zu diesem Vorstoss sei es aber «nicht zielführend, ein einseitiges Recht zu verankern. Bei sämtlichen Entscheiden müssen jeweils die Bedürfnisse beider Seiten berücksichtigt werden können» $3{ }^{1}$.

\section{Personalrecht von Kantonen}

19 Die Vielfalt von Bestimmungen in den kantonalen Personalgesetzen kann hier nicht vollständig, sondern nur typisierend behandelt werden. Bei den meisten Regelungen geht es um die Verbesserung der Vereinbarkeit von Beruf und Fami$\operatorname{lie}^{32}$, bei einzelnen um die Reduktion wegen der Ausübung eines öffentlichen Amtes33, um Massnahmen bei Stellenabbau 34 oder um schrittweise Pensionierung35. Nur wenige Regelungen nehmen hingegen keinen Bezug auf eine spezifische Situation 36 .

31 Stellungnahme vom 17. August 2016. Die Motion wurde am 14. Dezember 2017 durch den Nationalrat mit 54 zu 136 Stimmen abgelehnt (AB 2017 N 2174).

32 Überblick zu den kantonalen Regelungen des unbezahlten Urlaubs für Mütter, Väter und Eltern bei Kurt Pärli, Eltern im Arbeitsverhältnis, in: FamPra.ch 2016, S. 933-956, 952.

33 Z.B. Art. 119 Abs. 3 Gesetz über das Staatspersonal des Kantons Freiburg.

34 Z.B. § 7 Verordnung über die Massnahmen bei einem grösseren Stellenabbau (Sozialplan) des Regierungsrates Luzern.

35 Z.B. Art. 27a Gesetz betreffend die Besoldung der Angestellten des Staates Wallis, wonach der Staatsrat in den letzten fünf Jahren vor Erreichung der statutarischen Alterslimite eine Reduktion des Pensums gewähren kann. Für Lehrkräfte gibt es in vielen Kantonen Regelungen zur sog. Altersentlastung (z.B. § 38 Verordnung des Regierungsrats Thurgau über die Rechtsstellung der Lehrpersonen an den Volksschulen).

36 Z.B. § 31 Verordnung über die Arbeitsverhältnisse des Staatspersonals (Personalverordnung) des Kantons Schaffhausen, wonach Teilzeitstellen
20 Materiell zeigt sich ein Spektrum, welches von reinen Absichtserklärungen, Teilzeit zu fördern37, über KannVorschriften für Pensumsreduktionen 38 bis zum durchsetzbaren Anspruch auf Verkürzung39 und auf Wiederaufstockung des Arbeitspensums ${ }^{40}$ reicht. Teilweise wird explizit erwähnt, dass durch Teilzeitbeschäftigung weder die Aufgabenerfüllung noch der Betriebsablauf behindert werden darf 41 .

\section{Gesamtarbeitsverträge}

21 Ein Blick in Gesamtarbeitsverträge ist wenig inspirierend. Das Bedürfnis von Arbeitnehmenden nach Veränderung des Arbeitspensums scheint kaum in die Vertragsverhandlungen einzufliessen. Im GAV Swissmem findet sich im Art. 26 unter dem Titel «Vereinbarkeit von Beruf und Privatleben» eine Empfehlung an die Firmen, «bei der Arbeits- und Arbeitszeitgestaltung nach den betrieblichen Möglichkeiten auf die Bedürfnisse der Arbeitnehmenden, insbesondere derjenigen mit Familienpflichten, besonders

eingerichtet werden können, aber kein Anspruch auf Änderung des Beschäftigungsgrads besteht.

37 Z.B. § 5 Abs. 2 lit. c Personalgesetz Basel-Stadt, wonach flexible Arbeitszeitregelungen, insbesondere die Schaffung von Teilzeitarbeit in allen Bereichen und auf allen hierarchischen Stufen gefördert werden soll.

38 Gem. § 96 Abs. 3 Vollzugsverordnung zum Personalgesetz Zürich kann Müttern nach dem Mutterschaftsurlaub auf Gesuch hin der Beschäftigungsgrad reduziert werden, soweit die dienstlichen Verhältnisse es zulassen.

39 Gem. Art. 6oc Personalverordnung Kanton Bern gilt die gleiche Regelung wie beim Bundespersonal nach Art. 60a BPV.

40 Gemäss Art. 4A Abs. 4 RPAC haben Arbeitnehmende des Kantons Genf Anspruch auf 2 Jahre Elternurlaub bzw. alternativ bei Einverständnis der Vorgesetzten Anrecht auf Pensumsreduktion und danach das Recht auf Rückkehr in die frühere Funktion ohne Verlust der Rechte wie Lohnerhöhung und Dienstaltersanstieg.

41 Z.B. § 3 Abs. 1 Personalgesetz des Kantons Zug. 
Rücksicht zu nehmen.» Einzig der Firmenvertrag der Metron $\mathrm{AG}$ räumt in Art. 20 Müttern und Vätern anstelle des unbezahlten Urlaubs von 8 Monaten (im Anschluss an den viermonatigen Mutterschaftsurlaub) das Recht ein, die Arbeitszeit zu reduzieren. Der Anspruch besteht, «bis die Reduktion kapazitätsmässig 8 Monate beträgt».

\section{Vorstösse zur Änderung des Obligationenrechts}

\section{a) Anspruch für alle Arbeitnehmenden}

Nationalrat Jean-Claude Rennwald wollte 2006 mit dem Postulat «Freie Wahl der Arbeitszeit»42 den Bundesrat beauftragen, «konkrete Massnahmen zu prüfen, damit Erwerbstätige bei der freien Wahl von Arbeitszeit und Arbeitszeitmodell unterstützt werden. (...) Die Arbeitgeber sollen deshalb dazu verpflichtet werden, mit allen Arbeitnehmerinnen und Arbeitnehmern, die ihre Arbeitszeit und/oder ihr Arbeitszeitmodell nach oben oder nach unten anpassen wollen, zumindest ein Gespräch zu führen. Die Ablehnung eines solchen Antrags muss gegebenenfalls schriftlich begründet werden». Das Geschäft wurde auf Antrag des Bundesrates abgeschrieben. Er verwies auf die Vertrags(änderungs)freiheit unter Beachtung der zwingenden Bestimmungen und auf die Gerichtspraxis, wonach Betriebe das Betriebsrisiko nicht auf die Arbeitnehmenden verlagern dürfen. Das Postulat sei abzulehnen, da bereits «das geltende Recht verhindert, dass der Arbeitgeber die gewünschte

42 Gesch.-Nr. 06.3531.
Flexibilität dazu benützt, die Arbeitszeit einseitig festzulegen» 43 .

23 Mit einem weiteren Postulat wollte Nationalrat Manuel Tornare den Bundesrat beauftragen zu prüfen, "welche gesetzgeberischen Schritte notwendig sind, um die Arbeitsplatzteilung in der Schweiz zu etablieren»44. Wie auch bezüglich eines ähnlichen Postulats von Nationalrätin Géraldine Marchand-Balet45 sah der Bundesrat keinen Handlungsbedarf: «Primär ist es Aufgabe der Privatwirtschaft, optimale Arbeitsbedingungen zu entwickeln und umzusetzen». Anpassungen auf gesetzlicher Ebene seien nicht erforderlich 46 .

\section{b) Anspruch für Arbeitnehmende mit Kindern}

24 Auch der Parlamentarischen Initiative von Nationalrätin Nadine Masshardt «Vereinbarkeit von Beruf und Familie verbessern; Rechtsanspruch auf Reduktion des Beschäftigungsgrades nach der Geburt»47 war kein Erfolg beschieden. Sie zielte darauf ab, die Regelung von Art. 60a BPV48 mit einer Ergänzung des Obligationenrechts auf die privatrechtlichen Arbeitsverhältnisse zu übertragen, wobei kleinere Unternehmen ausgenommen worden wären. Das Anliegen scheiterte jedoch im Nationalrat 49 u.a. mit folgenden Argumenten: «Flexibilität

43 Stellungnahme des Bundesrates vom 29. November 2006. Das Geschäft wurde 2009 abgeschrieben, weil es seit mehr als zwei Jahren hängig war.

44 Gesch.-Nr. 17.3936.

45 Gesch.-Nr. 17.3307.

46 Antwort des Bundesrats vom 1. Dezember 2017.

47 Gesch.-Nr. 15.470.

48 Oben Rz. 16.

49 Mit $67 \mathrm{zu} 110$ Stimmen bei 3 Enthaltungen scheiterte das Geschäft in der Debatte am 1. Juni 2017 (AB 2017 N 883). 
ist bei gutem Willen immer möglich und geradezu ein Gebot der Stunde ... Angesichts der Vielzahl an flexiblen Arbeitszeitmodellen besteht auch kein Bedürfnis nach einem staatlichen Eingriff»50. Und der Sprecher der Kommissionsmehrheit verwies auf die Vertragsfreiheit und erklärte zudem: «cette proposition aurait, ..., le gros désavantage de tuer, dans une certaine mesure, la discussion qui doit avoir lieu»51. Hier sei der Hinweis erlaubt, dass in Deutschland die gesetzliche Verankerung eines Teilzeitanspruchs die betriebsinternen Diskussionen über Arbeitspensen keineswegs abgetötet, sondern diese vielmehr belebt hat ${ }^{2}$.

Nationalrätin Rebecca Ana Ruiz machte mit einer Interpellation auf das Problem «Mutter werden und dann den Job verlieren?»53 aufmerksam. Zudem verlangte Nationalrätin Silvia Schenker mit der Interpellation «Kündigung nach Mutterschaftsurlaub»54 vom Bundesrat Antworten insbesondere zur Frage, welche Möglichkeiten bestehen um $\mathrm{zu}$ verhindern, dass Frauen kurz nach dem Mutterschaftsurlaub «unter irgendeinem Vorwand» gekündigt werde, obwohl dies gegen das Gleichstellungsgesetz verstosse. Diese Rechtsauffassung teilte der Bundesrat in seiner Antwort. Er bestätigte, dass das «Problem weiterhin besteht» und nach Schweizer Recht auch eine unrechtmässige Kündigung das Arbeitsverhältnis beendet55. Eine Vorlage mit schärferen Sanktionen (Erhöhung der Entschädigung) sei aber sistiert worden. Über strengere Sanktionen hinaus sei

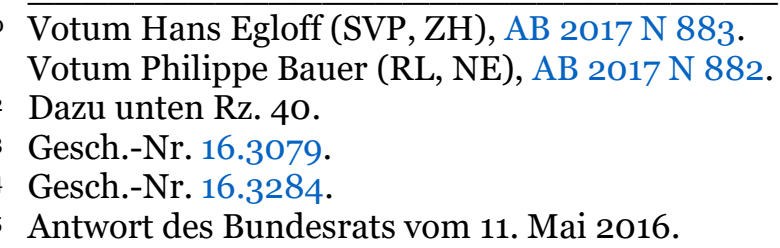

nach wie vor ein Umdenken erforderlich: «Die Vorteile und der positive Beitrag arbeitstätiger Mütter müssen anerkannt werden». Der Bund unterstütze daher Unternehmen bezüglich familienfreundlicheren Arbeitsbedingungen.

26 Auch Nationalrätin Regula Rytz wies auf eine Zunahme von Arbeitskonflikten rund um die Mutterschaft hin und verlangte mit einem Postulat vom Bundesrat einen «Bericht über die Diskriminierung von Eltern auf dem Arbeitsmarkt und ein(en) Aktionsplan als Gegenstrategie»56. In seiner Antwort bedauerte der Bundesrat einmal mehr die gesetzeswidrigen Praktiken, sah aber aufgrund der «laufenden Bestrebungen» keinen Bedarf für weitere Massnahmen57.

\section{c) Anspruch für Arbeitnehmende mit Betreuungsaufgaben}

27 Ende Dezember 2014 publizierte der Bundesrat seinen Bericht «Unterstützung für betreuende und pflegende Angehörige» und zeigte aufgrund seiner Situationsanalyse und einem Vergleich mit ausländischen Regelungen58 den Handlungsbedarf auf. Der Aktionsplan des Bundes sieht nun folgende Massnahmen vor: Erlass einer rechtlichen Grundlage für einen Betreuungsurlaub mit oder ohne Lohnfortzahlung - oder alternative Unterstützungsmöglichkeiten für längere pflegebedingte Abwesenheiten, sowie «Prüfung» eines Kündigungsschutzes

56 Gesch.-Nr. 16.3589.

57 Stellungnahme des Bundesrats zu beiden Geschäften vom 31. August 2016.

58 Informative Übersicht auf S. 42 des bundesrätlichen Berichtes «Unterstützung für betreuende und pflegende Angehörige; Situationsanalyse und Handlungsbedarf für die Schweiz», Bern 2014. 
während des Betreuungsurlaubs59. Eine entsprechende Gesetzesvorlage sollte demnächst in die Vernehmlassung gehen ${ }^{60}$.

\section{Rechtslage im Ausland}

28 Bei politischen Vorstössen zum Ausbau der Zeitsouveränität von Arbeitnehmenden wird verschiedentlich auf ausländisches Recht verwiesen. Diesen Regelungen und den Erfahrungen damit soll im Folgenden nachgegangen werden.

\section{Europäische Union}

29 Die Änderung von Arbeitspensen wird im Recht der Europäischen Union hauptsächlich in der Richtlinie 97/81/EG und dem dazugehörenden Anhang «Rahmenvereinbarung über Teilzeit» ${ }^{61}$, aber auch in der Elternzeitrichtlinie 2010/18/EU62 angesprochen.

30 In $\S 5$ der Rahmenvereinbarung über Teilzeit wird an den Grundsatz der Nichtdiskriminierung von Teilzeit- und Vollzeitbeschäftigten erinnert. In diesem Sinne werden die Mitgliedsstaaten und die Sozialpartner aufgefordert, Hindernisse, die die Teilzeitarbeitsmöglichkeiten beschränken können, zu beseitigen (Ziff. 1). Weigern sich Arbeitnehmende

Bericht des BR (Fn. 58), S. 39; Massnahmen 4A und $4 \mathrm{~B}$.

60 Gemäss Angaben der Bundesverwaltung ist die Vernehmlassung im Zeitraum Juli bis Oktober 2018 geplant.

61 RL vom 15. Dezember 1997 zu der von UNICE, CEEP and EGB geschlossenen Rahmenvereinbarung über Teilzeitarbeit (ABl. Nr. L 14 vom 20. Januar 1998 S. 9-14); L 283/36.

62 Richtlinie 2010/18/EU vom 8. März 2010 zur Durchführung der von BUSINESSEUROPE, UEAPME, CEEP und EGB geschlossenen überarbeiteten Rahmenvereinbarung über den Elternurlaub (ABl. EG Nr. L 68 vom 18. März 2010, S. 1320). einer Änderung ihres Arbeitspensums zuzustimmen, kann ihnen aus diesem Grund nicht gültig gekündigt werden (Ziff. 2). Zudem sollten Arbeitgebende «soweit dies möglich ist» Änderungswünsche ihrer Arbeitnehmenden berücksichtigen, über entsprechende Stellenangebote rechtzeitig informieren und den Zugang zu Teilzeitarbeit «auf allen Ebenen des Unternehmens» erleichtern (Ziff. 3).

31 Gemäss der Rahmenvereinbarung zur Elternzeitrichtlinie haben die Mitgliedsstaaten bzw. die Sozialpartner einen mindestens vier Monate dauernden Elternurlaub als Vollzeit- oder Teilzeiturlaub einzurichten (§ 2 und 3). Sie dürfen die Modalitäten und Bedingungen für die Inanspruchnahme regeln unter Berücksichtigung der erlassenen Mindestvorschriften. Dazu gehören etwa das Recht, im Anschluss an den Elternurlaub ohne Rechtsnachteile an den früheren $\mathrm{Ar}$ beitsplatz zurückzukehren ( $§ 5$ ). Es sollte auch sichergestellt werden, dass bei der Rückkehr aus dem Elternurlaub Änderungen der Arbeitszeiten für eine bestimmte Dauer unter Berücksichtigung der Bedürfnisse beider Parteien ermöglicht werden (§ 6).

\section{Deutschland}

32 Die Bundesrepublik Deutschland hat die Vorgaben des EU-Rechts mit dem Gesetz über Teilzeitarbeit und befristete Arbeitsverträge (Teilzeit- und Befristungsgesetz, TzBfG) und dem Gesetz zum Elterngeld und zur Elternzeit (Bundeselterngeld- und Elternzeitgesetz, BEEG) umgesetzt. Zudem haben Schwerbehinderte gem. § 164 Abs. 5 Sozialgesetzbuch IX einen Anspruch auf Verkürzung der 
Arbeitszeit, wenn dies wegen der Art und Schwere der Behinderung notwendig ist.

Mit $\S 8$ TzBfG erhielten 2001 die Arbeitnehmenden in deutschen Betrieben mit mehr als 15 Mitarbeitenden unter Einhaltung von bestimmten Fristen einen Anspruch auf Verringerung der Arbeitszeit und auf Änderung ihrer Verteilung. Konnte durch die gesetzlich verlangte Erörterung einer Vereinbarung keine einvernehmliche Anpassung erzielt werden, hat der Arbeitgeber dem Gesuch zuzustimmen, soweit betriebliche Gründe nicht entgegenstehen. Ein betrieblicher Grund liegt insbesondere vor, wenn die Verringerung der Arbeitszeit «die Organisation, den Arbeitsablauf oder die Sicherheit im Betrieb wesentlich beeinträchtigt oder unverhältnismässige Kosten verursacht». Nach der Rechtsprechung genügt es, wenn Arbeitgebende rational nachvollziehbare, hinreichend gewichtige Gründe beweisen, allerdings kann die Ablehnung nicht allein mit den unternehmerischen Vorstellungen von der «richtigen» Arbeitszeitverteilung begründet werden63. Dem Anspruch von Eltern auf Verringerung der Arbeitszeit aufgrund von $\S 15$ BEEG können Arbeitgebende nur «dringende» betriebliche Gründe entgegensetzen.

34 Aufgrund von §9 TzBfG haben Arbeitnehmende zwar kein Recht auf Erhöhung des Arbeitspensums, aber einen Anspruch auf bevorzugte Berücksichtigung, wenn das entsprechende Arbeitsvolumen vorhanden ist 64 . Aufgrund des aktuellen

63 Silke Clasvorbeck, Der Anspruch auf Verringerung und Verlängerung der Arbeitszeit, in: AuR 3/2018, S. 116-119, 117.

64 Rainer Schlegel, Arbeitszeitsouveränität - sozialrechtliche Aspekte, in: AuR 7/2016, S. 268-273, 269.
Koalitionsvertrags soll in naher Zukunft die «Teilzeitfalle» behoben werden, indem der Aufstockungsanspruch mit einer Beweislastumkehr erleichtert wird ${ }^{65}$.

\section{Niederlande}

35 Die Niederlande weisen schon länger den weitaus höchsten Anteil von Teilzeitbeschäftigten im europäischen Vergleich aus $^{66}$. Bereits per 1. Juli 2000 haben die Niederlande die EU-Teilzeit-Richtlinie mit einem Rechtsanspruch auf Teilzeitarbeit im Wet Aanpassing Arbeidsdur (WAA) umgesetzt. Der Anspruch auf Teilzeitarbeit besteht nach einem Jahr Beschäftigungsdauer in Betrieben mit mehr als 10 Arbeitnehmenden. Die gewünschte Reduktion des Arbeitspensums kann nur aus «schwerwiegenden betrieblichen Gründen» abgelehnt werden ${ }^{67}$. Zudem gilt bislang allein in den Niederlanden ein explizites Recht auf Erhöhung der vertraglichen Arbeitszeit68.

\section{4. Übrige europäische Länder}

36 Einen durchsetzbaren Anspruch auf einen Wechsel von Vollzeit- zu Teilzeitarbeit gibt es in einigen Ländern Europas, meist ist er jedoch an bestimmte Krite-

65 Annelie Buntenbach, Arbeitsrecht - Streitpunkt aller Verhandlungen, in: AuR 3/2018, S. 109.

66 Nämlich 46,6 \% gem. Eurostat, Beschäftigungsstatistik.

67 Ralph Kattenbach, Der Teilzeitanspruch in der betrieblichen Anwendung, Eine Analyse zum Einfluss von Personalverantwortlichen auf die betriebliche Umsetzung des Teilzeitanspruches, Diss. Hamburg, München 2009, S. 33.

68 Jörg Flecker/Annika Schönauer/Christoph Hermann/Bernadette Allinger, Arbeitszeitverkürzung zur Umverteilung von Arbeit, Internationale Beispiele, Bericht an das Bundesministerium für Arbeit, Soziales und Konsumentenschutz, Wien 2009, S. 14. 
rien (Unternehmensgrösse ab $10^{69}$ bis 2070 Arbeitnehmende) gebunden oder auf besondere Zielgruppen beschränkt. Die häufigste Zielgruppe sind Eltern71, z. T. aber auch erkrankte ${ }^{72}$ und73/oder ältere Mitarbeitende74 oder Arbeitnehmende mit spezifischen Betreuungspflichten wie Pflege von Familienangehörigen oder Sterbebegleitung75. Die Ansprüche sind in der Regel nicht durchsetzbar, wenn das Unternehmen betriebliche Gründe vorbringt und beweisen

$69 \overline{\text { Z.B. haben in Belgien Unternehmen mit weniger }}$ als 10 Mitarbeitenden Beweiserleichterungen bezüglich der negativen wirtschaftlichen Folgen (Flecker u.a. Fn. 68, S. 16).

70 Auf der anderen Seite findet sich Österreich, welches relativ restriktive Teilzeitansprüche nur gegenüber Privatbetrieben mit mehr als 20 Arbeitnehmenden kennt ( $\$ 15 \mathrm{~h}$ Mutterschutzgesetz und $\S 8$ Väter-Karenzgesetz).

${ }^{71}$ Z.B. besteht gem. französischem Code du travail Art. L.1225-47 im Anschluss an den Mutterschaftsurlaub ein Anspruch auf Elternurlaub oder Teilzeitbeschäftigung mit mind. 16 Wochenstunden.

72 Einen Anspruch auf Teilzeitarbeit haben z.B. in Italien seit 2003 Beschäftigte mit Krebsleiden (Kerstin Albers, Rechtsanspruch auf Verringerung der Arbeitszeit in Deutschland und Italien: eine rechtsvergleichende Studie, Diss. Bremen, Baden-Baden 2010, S. 23).

73 Einen Anspruch auf Reduzierung der Arbeitszeit haben nach norwegischem arbeidsmiljøloven $\S 10-2$ (3) Arbeitnehmende, ab 62. Altersjahr oder solche, die durch gesundheitliche, soziale oder andere gewichtige Wohlfahrtsgründe einen Bedarf haben, sofern dies ohne wesentliche Nachteile für das Unternehmen umgesetzt werden kann (Annemarie Aumann/Melanie Hack, Wahlarbeitszeit und Arbeitszeitflexibilisierung ; Modelle einer selbstbestimmten Erwerbsbiografie in Deutschland und Norwegen, in: Zesar 7/2016, S. 266-276, 272.

74 In Belgien besteht z.B. ein Anspruch auf Reduktion der Arbeitszeit um 50 \% oder 1/5 für Beschäftigte mit einem Mindestalter von 50 Jahren, die mindestens 20 Jahre beschäftigt waren (Flecker u.a. (Fn. 68), S. 16).

75 So besteht etwa in Österreich gem. § 14a ff. AVRAG ein Herabsetzungsanspruch für Sterbebegleitung, Betreuung schwersterkrankter Kinder oder Pflege naher Familienangehöriger. kann $^{76}$. Teilweise wirken auch komplizierte Verfahrensvorschriften prohibitiv77. Gelegentlich werden Ansprüche auf Reduktion des Pensums mit Lohnersatzleistungen verbunden 78 , sind arbeitsmarktpolitisch motiviert79 oder mit kollektiven Verträgen gekoppelt ${ }^{80}$.

37 Für die Erhöhung des Arbeitspensums sehen einige nationale Rechtsordnungen eine bevorzugte Behandlung von bereits beschäftigten Teilzeit-Arbeitnehmenden vor $^{81}$. In vielen Ländern finden sich Bestimmungen, die Kündigungen von Arbeitnehmenden als unwirksam erklären, welche eine Arbeitszeitänderung gewünscht haben 82 .

76 Z.B. Art. L3123-26 des Code du travail von Frankreich: «...s'il peut démontrer que le changement d'emploi demandé aurait des conséquences préjudiciables à la bonne marche de l'entreprise».

77 Für Österreich: Franz Marhold/Michael Friedrich, Österreichisches Arbeitsrecht, 2. Aufl., Wien 2012, S. 99. Für Italien: Albers (Fn. 72), S. 182.

78 So wird in Dänemark und Portugal für unfreiwillige Teilzeit eine Entschädigung der Arbeitslosenversicherung bezahlt (Flecker u.a. Fn. 68, S. 13).

79 Nach österreichische Arbeitslosenversicherungsgesetz (§ 26a) wird z.B. für Bildungsteilzeit (gem. $\S$ 11a Arbeitsvertragsrechts-Anpassungsgesetz) ein Bildungsteilzeitgeld ausgerichtet.

8o Eine kollektive betriebliche Solidaritätsvereinbarung ist u.U. Voraussetzung für eine Herabsetzung der Arbeitszeit (Luca Nogler, Arbeitszeitregelung in Italien, in: Geiser/Müller (Hrsg.), Arbeitszeiten in Europa, Zürich 2016, S. 113). S. auch im französischen Code du travail Art. L2241-13.

81 So z.B. in Schweden und Frankreich (Flecker u.a. Fn. 68, S 14) und mit differenzierten Regeln in Spanien (Vicente Calle/Stefanie Prehm, Arbeitsrecht in Spanien, in: Henssler/Braun (Hrsg.), Arbeitsrecht in Europa, 3. Aufl., Köln 2011, Rz. 108 ff.).

82 Nach britischem Recht gelten z.B. Kündigungen wegen einer Anfrage auf variable Arbeitszeiten oder im Zusammenhang mit Teilzeitbeschäftigung als «unfair dismissal» (Beatrix Tröger/ Nadja Ross-Kirsch, Arbeitsrecht in Grossbritannien, Praxisrelevante Regelungen und Unterschiede zur deutschen Rechtslage, 2. Aufl., Wiesbaden 2017, S. 128). 


\section{Erfahrungen mit} Anpassungsansprüchen

38 Über die unterschiedlichen nationalen Ausgestaltungen hinaus interessieren insbesondere die Erfahrungen, welche Deutschland und Holland mit ihren am stärksten verankerten Änderungsrechten für die Arbeitnehmenden gemacht haben.

39 In Deutschland erhofften sich Befürworter des Teilzeitanspruchs beschäftigungswirksame Effekte auf den Arbeitsmarkt. Gegner rechneten hingegen mit einer Flut von Anträgen und mit massiven organisatorischen und wirtschaftlichen Problemen für die Unternehmen. Allerdings erweist sich nun die Umsetzung als unspektakulär. Die Rechtsnormen werden von Unternehmen in Deutschland und in den Niederlanden nicht als problematische Vorschriften beurteilt ${ }^{83}$. Sie sehen Teilzeitbeschäftigung als Mittel, um gutes Personal halten zu können, welches im gewünschten Pensum auch motivierter arbeitet. Sie erwähnen aber auch die erhöhten Anforderungen an eine gute Personaleinsatzplanung und höhere Kosten ${ }^{8}$.

Die Einführung des Anspruchs beeinflusste in Deutschland die schon zuvor langsam wachsende Teilzeitquote nicht wesentlich, dennoch ist es dem Gesetzgeber offenbar gelungen, den betrieblichen Einsatz von Teilzeitbeschäftigten zu stimulieren. Die Gesetze scheinen insbesondere in jenen Unternehmen gewirkt zu haben, die vor deren Einführung keine Teilzeitbeschäftigten eingesetzt hatten 85 .

\footnotetext{
83 Kattenbach (Fn. 67), S. 237 bzw. S. 37 und 35.

84 Kattenbach (Fn. 67), S. 35 .

85 Hans-Dieter Gerner, Arbeitszeitverlängerung, Arbeitszeitkonten und Teilzeitbeschäftigung,
}

In Holland traf die Erwartung nicht ein, dass der gesetzliche Teilzeitanspruch zu einem Anstieg der Teilzeitquote von Männern führen werde ${ }^{86}$.

41 Weiterhin werden Teilzeit-Anträge in beiden Ländern sehr oft einvernehmlich geregelt. Streitigkeiten darüber werden in Deutschland nur selten vor Gericht getragen ${ }^{87}$. Auch in den Niederlanden waren drei Jahre nach Einführung des Anspruchs lediglich 22 Gerichtsfälle zu verzeichnen 88 . Arbeitnehmende stellen firmenintern Anträge auf Reduktion des Arbeitspensums ohnehin meist nur bei Aussicht auf Erfolg89, und es gibt Anzeichen dafür, dass sie die ablehnende Haltung von Vorgesetzten antizipieren ${ }^{90}$. Arbeitgebende können somit aufgrund der Machtasymmetrie den Änderungswunsch abwehren, ohne in die Situation zu kommen, betriebliche Gründe vorbringen und eine Interessenabwägung vornehmen zu müssen ${ }^{91}$.

42 Dennoch wird dem gesetzlichen Teilzeitanspruch Bedeutung zugemessen. Er benennt die Arbeitszeit als Verhandlungsgegenstand und stärkt allein dadurch die Verhandlungsposition der Arbeitnehmenden92. Gleichzeitig konnte auch festgestellt werden, dass die Gesetzestexte diejenigen Personalverantwortlichen un-

Ökonometrische Analysen, Diss. ErlangenNürnberg, Nürnberg 2009, S. 128.

86 Kattenbach (Fn. 67), S. 34. Auch in Belgien wurden die Rechte zu 78 \% von Frauen und zu $22 \%$ von Männern in Anspruch genommen, insbesondere von eher höher gebildeten Personen (Flecker u.a. Fn. 68 , S. 17).

87 So etwa Urteile des BAG vom 20. Januar 2015 (9 AZR 735/13 und 9 AZR 860/13).

88 Kattenbach (Fn. 67), S. 34.

89 Kattenbach (Fn. 67), S. 214.

90 Kattenbach (Fn. 67), S. 169.

91 Gerner (Fn. 85), S. 94.

92 Kattenbach (Fn. 67), S. 227. 
terstützen, welche Teilzeit-Arrangements intern rechtfertigen müssen, obgleich Teilzeitbegehren in den Unternehmen nicht allein nach dem Gesetz, sondern stark nach eigenen Gerechtigkeitsvorstellungen der Vorgesetzten beurteilt werden93.

\section{Lösungsansätze im}

\section{schweizerischen Privatrecht}

\section{Ausgangslage}

43 Die Analyse der heutigen Rechtslage bei Änderungswünschen 94 hat deutlich gemacht, dass für Arbeitnehmende die Realisierung einer Veränderung der ursprünglich vereinbarten Arbeitszeit mit höheren Risiken verbunden ist als für die Arbeitgebenden. Dies ist problematisch, denn ein synallagmatisches Vertragsverhältnis sollte sich durch eine grundsätzlich äquivalente Risikoverteilung auszeichnen95. Zudem fällt bei Arbeitsverträgen ins Gewicht, dass änderungswillige Arbeitnehmende i.d.R. ein grösseres Interesse am Weiterbestand des Vertrags haben als ihre Arbeitgebenden ${ }^{96}$.

44 Verschiedene Vorstösse zur Änderung des Obligationenrechts sind bisher gescheitert97. Daher soll zunächst geprüft werden, ob die Situation ohne Gesetzesrevision, d.h. durch die Rechtsprechung

93 Kattenbach (Fn. 67), S. 168 und 214.

94 Oben Rz. 12 ff.

95 Felix Buff, Vertragliche Anpassungsklauseln im schweizerischen Recht, Diss. Zürich, Zürich 2016, S. 37.

96 Buff (Fn. 95), S. 72; Adrian von Kaenel, Einseitige Vertragsänderungsrechte des Arbeitgebers, in: Wyler/Meier/Marchand (Hrsg.), Regards croisés sur le droit du travail: Liber Amicorum pour Gabriel Aubert, Genf 2015, S. 331-345, 337.

97 Oben Rz. 22 ff. aufgrund der heutigen Rechtslage entschärft werden könnte.

\section{Rechtsprechung}

\section{a) Anspruch aufgrund der Fürsorgepflicht}

45 Wie bereits ausgeführt riskieren Unternehmen, welche Präventionspflichten vernachlässigen und Reduktionswünsche wegen zu hoher Arbeitsbelastung von Arbeitnehmenden ablehnen, für daraus entstandene Schäden wegen Verletzung der Fürsorgepflicht zu haften 98 .

46 Anders ist die Rechtslage, wenn der Grund für den Änderungswunsch nicht in einer Pflichtverletzung der Arbeitgeberseite liegt, sondern mit der Lebenssituation von Arbeitnehmenden zusammenhängt. Die Frage, ob sich in diesen Fällen aus dem Persönlichkeitsschutz (Art. 328 OR) ein Recht von Arbeitnehmenden ableiten lässt, den Beschäftigungsgrad zu reduzieren oder zu erhöhen, wird in der Lehre meist klar verneint, weil die Änderung eines wesentlichen Vertragsinhaltes des Konsenses bedürfe 99 .

47 Einen Ansatzpunkt für Arbeitnehmende mit familiären Betreuungspflichten könnten allerdings Art. 8 Ziff. 1 EMRK und Art. 13 BV bieten. Das Recht auf Achtung des Privat- und Familienlebens ist als Grundrecht (gegenüber dem Staat) konzipiert; eine Drittwirkung gegenüber einem privaten Arbeitgeber wird nicht

98 Oben Rz. 11.

99 Hensch (Fn. 18), S. 1642; Gabriela Riemer-Kafka, Einseitige Arbeitszeitveränderungen durch den Arbeitgeber, in: AJP 2017, S. 312-326, 320. Nicht kategorisch ablehnend: Pärli (Fn. 32), S. 945. 
anerkannt ${ }^{100}$. Hingegen haben Gerichte dafür zu sorgen, «dass die Grundrechte, soweit sie sich dazu eignen, auch unter Privaten wirksam werden» (Art. 35 Abs. 3 BV). In der schweizerischen Rechtsprechung ist das Prinzip der Vertragsfreiheit jedoch eine derart «tief eingegrabene Denkkategorie»101, dass es gegenüber solchen Ansätzen eine kaum zu überwindende Hürde darstellt. Es ist daher unrealistisch, über ein Gerichtsverfahren mit einer solchen Auslegung der Fürsorgepflicht ein Recht auf Weiterführung eines Arbeitsverhältnisses mit verändertem Pensum zu erreichen.

\section{b) Teilkündigung}

48 Grundsätzlich hätten Arbeitnehmende das Recht, eine Änderungskündigung auszusprechen in dem Sinne, dass sie ihr Arbeitsverhältnis kündigen für den Fall, dass das Unternehmen eine Weiterführung mit verändertem Pensum ableh-

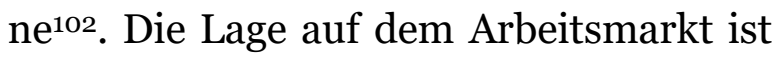
allerdings kaum je so, dass Arbeitnehmende auf diesem Weg den Änderungswunsch ohne Verlust des Arbeitsplatzes durchsetzen können.

49 Etwas anders lägen die Verhältnisse, wenn Arbeitnehmende das Recht beanspruchen könnten, einen Teil des Pensums zu kündigen, so dass das Arbeitsverhältnis mit reduzierter Arbeitszeit (und entsprechend gekürztem Lohn) weiterläuft. Eine Teilkündigung des Arbeitsverhältnisses als bedingtes Gestaltungsrecht wird durch die schweizerische

\footnotetext{
100 Von Kaenel (Fn. 96), S. 340 f.

101 Martin Farner, Der Aufhebungsvertrag, in: ArbR 2010, S. 33-52, 35.

102 Witzig (Fn. 1), S. 178.
}

Rechtsprechung ${ }^{103}$ und Lehre aber abgelehnt, sofern nicht etwas anderes vereinbart wurde ${ }^{104}$. Eine Teilkündigung müsse als Offerte für eine Vertragsänderung verstanden werden ${ }^{105}$. Dazu gibt es aber differenziertere Haltungen ${ }^{106}$, wonach eine Teilkündigung nur dann abzulehnen ist, wenn sie das vereinbarte Ordnungsund Äquivalenzgefüge eines Vertrags stört, denn im Allgemeinen entspreche Kontinuität den Interessen der Vertragsparteien eher als Diskontinuität, insbesondere im Arbeitsverhältnis ${ }^{107}$. Dies gilt ganz besonders dann, wenn ein sachlicher Grund für die Teilkündigung vorliegt ${ }^{108}$.

5o Schweizerische Gerichte mussten zu einer Teilkündigung des Arbeitspensums durch Arbeitnehmende bisher noch nie explizit Stellung nehmen. Es fragt sich aber ohnehin, ob Arbeitnehmende damit viel gewonnen hätten, solange das mit dem reduzierten Pensum weiterlaufende

103 Urteil des Bundesgerichts 4C.398/2001 vom 6. Juni 2002, E. 2.2.1.; hingegen erachtete die Personalrekurskommission des Kantons BaselStadt eine Teilkündigung des Arbeitspensums eines Museumsaufsehers seitens des Arbeitgebers als zulässig (BJM 2015, S. 289).

104 Marco Kamber, Die Änderungskündigung im Arbeitsvertragsrecht; Insbesondere die Rechtsprechung zur Missbräuchlichkeit, Diss. Basel, Bern 2014, Rz. 217; Manfred Rehbinder/JeanFritz Stöckli, Berner Kommentar zu den Art. 31933ob OR, 2. Aufl., Bern 2014, Art. 335 OR N 5.

105 Adrian Staehelin, Der Arbeitsvertrag, Zürcher Kommentar, 4. Aufl., Zürich 2014, Art. 335 OR N 8.

106 Im Ansatz etwa BGE 102 II 376, E.4, wonach je nach Gestaltungsrecht und Interessenlage des einzelnen Falles zu unterscheiden ist. Andreas von Tuhr/Arnold Escher, Allgemeiner Teil des Schweizerischen Obligationenrechts, Zürich 1974, S. 49, erachten die Teilkündigung eines Gläubigers als zulässig, wenn er befugt ist, einen Teil der Leistung zu verlangen.

107 Angie Schneider, Vertragsanpassung im bipolaren Dauerschuldverhältnis, Habil. Köln, Tübingen 2016, S. $292 \mathrm{f}$.

${ }_{108}$ Schneider (Fn. 107), S. 295. 
Arbeitsverhältnis jederzeit durch die Arbeitgeberseite gekündigt werden kann. Die Missbräuchlichkeit einer solchen Kündigung wäre zwar leichter zu beweisen, aber der Verlust der Stelle wäre nicht zu verhindern.

\section{c) Sachlicher Kündigungsschutz}

51 Wird das Arbeitsverhältnis gekündigt, nachdem Arbeitnehmende eine Änderung des Arbeitspensums gewünscht haben, können sie die Kündigung zwar anfechten mit der Begründung, sie hätten nach Treu und Glauben einen «Anspruch aus dem Arbeitsverhältnis» geltend gemacht (Art. 336 Abs. 1 lit. d OR). Nach strenger Auslegung des Wortlauts müsste ein Gericht dieser Ansicht aber nicht folgen, da es bislang kein gesetzliches Recht auf Pensumsänderung gibt. Es könnte allenfalls den Anspruch unter dem Aspekt des Persönlichkeitsschutzes (nach Art. 328 OR) beurteilen, indem eine sachlich nicht begründete Abweisung eines Änderungswunsches die Persönlichkeit von Arbeitnehmenden verletze ${ }^{109}$. Dieses Prozessrisiko wird jedoch kaum jemand auf sich nehmen, und Gerichte können schliesslich nur die Streitfragen beurteilen, welche an sie herangetragen werden.

Etwas besser sind die Voraussetzungen bei diskriminierenden Kündigungen rund um Mutterschaftt10. Obwohl mit Art. 9 GlG dafür eine Spezialnorm besteht, scheinen jedoch viele Betroffene den Gang an die Schlichtungsbehörden

109 Oben Rz. 46.

110 Zur Verbreitung dieses Phänomens: oben Rz. 25 f. Dennoch betreffen fast die Hälfte der auf www.gleichstellungsgesetz.ch erfassten Fälle Klagen wegen diskriminierender Kündigung. zu scheuen ${ }^{111}$. Immerhin sind Klagen wegen diskriminierender Kündigung relativ häufig von Erfolg gekrönt im Vergleich $\mathrm{zu}$ anderen Klagen nach GlG; sie werden nämlich in «nur» 56.8 Prozent der Fälle abgewiesen ${ }^{112}$.

\section{De lege ferenda}

53 Es führt offensichtlich kein Weg daran vorbei, das Arbeitsvertragsrecht zu revidieren, um paritätische Verhältnisse beim Wunsch nach Änderungen der Arbeitszeit herzustellen ${ }^{113}$.

54 Der Grundsatz der Vertragsfreiheit ermöglicht Vertragsparteien, ihre Rechtsbeziehungen selbstbestimmt zu regeln. Dass der Staat der Vertragsgestaltung und -beendigung keine Schranken setzt, lässt sich aber nur rechtfertigen, wenn zwischen den Vertragsparteien ausgeglichene Kräfteverhältnisse bestehen. Dies ist im Arbeitsverhältnis aber nicht der Fall, denn: «Der einzelne Arbeitnehmer ist typischerweise ungleich stärker auf sein Arbeitsverhältnis angewiesen als der Arbeitgeber auf den einzelnen Arbeitnehmer» ${ }^{114}$. Nimmt man Vertragsfreiheit und Flexibilisierungsbedürfnisse auf beiden Seiten ernst, kann man diese unterschiedlichen Rahmenbedingungen nicht ignorieren. Es gilt vielmehr, Voraussetzungen zu schaffen, dass beide Parteien einen möglichst gleich grossen und tatsächlich auch realisierbaren Entschei-

111 Dazu die Medienberichte, die in der Stellungnahme des Bundesrates zur Interpellation Schenker (Gesch.-Nr. 16.3248) erwähnt werden.

${ }_{112}$ Karine Lempen/Aner Voloder, Aktuelle Entwicklungen rund um das Gleichstellungsgesetz, in SJZ, S. 81-89, 86.

113 So auch die Ergebnisse der breit angelegten Studie von Kocher u.a. (Fn. 25), S. 337.

114 Urteil des Bundesverfassungsgerichts der BRD vom 23. November 2006 (1 BvR 1909/06), Rz. 52. 
dungsspielraum im Sinne einer materiellen Vertragsfreiheit haben ${ }^{115}$.

\section{a) Anspruch auf Änderung des Arbeitspensums}

55 Für eine Ergänzung ihres Vertragsrechts kann die Schweiz die bisher gemachten Erfahrungen in Ländern mit einem Anspruch auf Arbeitszeitreduktion nutzen ${ }^{116}$. Wie der knappe Überblick gezeigt hat, gibt es bereits verschiedene Ausgestaltungsmöglichkeiten, an denen sich die Schweiz orientieren kann. Eine vertiefte Analyse (die andernorts noch $\mathrm{zu}$ leisten ist), kann die Entscheidung ermöglichen, ob Änderungen der Arbeitszeit nach unten oder auch nach oben $\mathrm{zu}$ berücksichtigen sind, und klären, wie die Voraussetzungen seitens der Arbeitnehmenden wie beispielsweise Beschäftigungsdauer, Ankündigungsfristen, Dauer der Anpassung zu definieren sind.

Die Äquivalenz zwischen den Vertragsparteien lässt sich am besten erreichen, wenn der Anspruch auf Änderung des Arbeitspensums für alle Arbeitnehmenden gilt. Beschränkt man ihn auf Arbeitnehmende mit Betreuungspflichten, öffentlichen Ämtern, Weiterbildungsanliegen u. ä., hat dies den Nachteil, dass die Antragstellenden den Grund nennen und beweisen müssen, während Arbeitgebende ihrerseits Änderungen von einzelnen Arbeitspensen i.d.R. ohne Begründungspflicht umsetzen können. Die neuen Rechtsgrundlagen müssen aber andrerseits jene Gründe nennen, welche Unternehmen berechtigen, einen Änderungswunsch abzulehnen.

115 Andreas Abegg, Die zwingenden Inhaltsnormen des Schuldvertragsrechts, Diss. Fribourg, Zürich 2004, S. $106 \mathrm{ff}$.

116 Oben Rz. 28 ff..
57 Ein erster Schritt für den Ausbau der Zeitsouveränität von Arbeitnehmenden kann schon in absehbarer Zeit bei der angekündigten Vorlage für einen Betreuungsurlaub aufgrund des Aktionsplanes «Unterstützung für betreuende und pflegende Angehörige» getan werden ${ }^{117}$.

\section{b) Bestandesschutz}

58 Bei ungerechtfertigter Auflösung von Dauerschuldverhältnissen gilt grundsätzlich die Regel, dass die Kündigung bei Nichtvorliegen eines wichtigen Grundes unbeachtlich ist und das Vertragsverhältnis weiterhin besteht. Im schweizerischen Arbeitsvertragsrecht hat man jedoch «dogmatisch einen kühnen Schritt» gemacht ${ }^{118}$, indem die Sanktion für missbräuchliche und diskriminierende Kündigungen lediglich in einer finanziellen Entschädigung besteht (Art. 336a OR) und nicht in der Weiterführung des Arbeitsverhältnisses. Damit unterscheidet sich das schweizerische Recht von den anderen Rechtsordnungen in Europa ${ }^{119}$. Solange die Sanktionen so schwach bleiben, kann die Freiheit von Arbeitnehmenden im Hinblick auf Änderungswünsche nicht nachhaltig verbessert werden.

59 In Deutschland hat sich zudem gezeigt, dass es für Arbeitnehmende wichtig ist, dass sie während des Gerichtsverfahrens und der Prüfung der betrieblichen Gründe von den Unternehmen nur mit der beantragten reduzierten Arbeitszeit eingesetzt werden dürfen ${ }^{120}$, insbesondere wenn ihre Verpflichtungen ausserhalb des Arbeitsplatzes keinen Aufschub er-

\footnotetext{
117 Siehe oben Rz. 27.

118 Frank Vischer, Das schweizerische Arbeitsrecht: Rechtspolitische Weichenstellung, in: AJP 2013 S. 349-355, 351.

119 Rehbinder/Stöckli BK (Fn. 104), Art. 336 OR N 9. 120 Clasvorbeck (Fn. 63), S. 119.
} 
tragen, wie etwa bei Betreuung von schwer kranken Angehörigen.

60 Die Einführung eines effektiv durchsetzbaren Anspruchs auf Änderung des Arbeitspensums muss auch im schweizerischen Recht mit einem spezifischen Kündigungsschutz ergänzt werden. Denn eine solche Neuerung bliebe toter Buchstabe, wenn Arbeitgebende die Realisierung des Anspruchs mit einer Kündigung verhindern könnten und höchstens eine Entschädigung wegen missbräuchlicher Kündigung riskierten.

61 Eine wirksame Regelung könnte etwa so ausgestaltet werden: Haben Arbeitnehmende den Wunsch nach Veränderung des Pensums geäussert, kann das Arbeitsverhältnis von der Arbeitgeberin nicht gekündigt werden, solange das Verfahren für die Überprüfung der Ablehnungsgründe läuft und für eine bestimmte Zeit darüber hinaus (z.B. sechs Monate analog Art.10 GlG). Während dieser Sperrfrist dürfen die Arbeitnehmenden mit einem Reduktionswunsch nur im Umfang der beantragten Teilzeitstelle beschäftigt werden, wenn sie wichtige Gründe dafür geltend machen. In diesem Verfahrensstadium ist es zumutbar, dass Arbeitnehmende darlegen, aus welchem Grund sie das bisherige Arbeitspensums nicht mehr erbringen können.

\section{Schlussbetrachtung}

62 Die Vertragsparteien können im Verlauf eines Arbeitsverhältnisses aus unterschiedlichen Gründen den Wunsch haben, die vereinbarte Arbeitszeit nach oben oder nach unten anzupassen. Damit Arbeitnehmenden in gleichem Umfang wie die Arbeitgebenden dieses Anliegen realisieren können, ist eine Änderung des Arbeitsvertragsrechts erforderlich. Es geht dabei um die Realisierung von äquivalenteren Vertragsbeziehungen. Darüber hinaus hätte eine solche Revision den Effekt, dass gesellschaftlich erwünschte unbezahlte Arbeiten gewürdigt werden. 